Filippo Cademartiri

Erica Maffei

Alessandro Palumbo

Chiara Martini

Sara Seitun

Carlo Tedeschi

Roberto De Rosa

Teresa Arcadi

Ignazio Salamone

Alfredo Blandino

Annick C. Weustink

Nico R. Mollet

Pim J. De Feyter

Gabriel P. Krestin

Received: 12 March 2009

Accepted: 12 June 2009

Published online: 6 August 2009

(C) European Society of Radiology 2009

F. Cademartiri $\cdot$ E. Maffei $\cdot$

A. Palumbo - C. Martini - S. Seitun

Department of Radiology and

Cardiology, Azienda Ospedaliero-

Universitaria di Parma,

Parma, Italy

F. Cademartiri - A. Palumbo ·

C. Martini - A. C. Weustink .

N. R. Mollet - P. J. De Feyter

G. P. Krestin

Department of Radiology and

Cardiology, Erasmus Medical Center,

Rotterdam, The Netherlands

C. Tedeschi · R. De Rosa

Department of Radiology and

Cardiology, Ospedale San Gennaro,

Napoli, Italy

T. Arcadi · I. Salamone - A. Blandino

Department of Radiology,

University Hospital,

Messina, Italy

\section{Diagnostic accuracy of computed tomography coronary angiography in patients with a zero calcium score}

F. Cademartiri $(\bowtie)$

Department of Radiology,

Azienda Ospedaliero-Universitaria-Parma,

c/o Piastra Tecnica-Piano 0,

Via Gramsci, 14,

43100 Parma, Italy

e-mail: filippocademartiri@hotmail.com

Tel.: +39-521-703516

Fax: +39-521-703630

Abstract To evaluate the diagnostic accuracy of 64-slice CT coronary angiography (CT-CA) for the detection of significant coronary artery stenosis in patients with zero on the Agatston Calcium Score (CACS). We enrolled 279 consecutive patients (96 male, mean age $48 \pm 12$ years) with suspected coronary artery disease. Patients were symptomatic $(\mathrm{n}=208)$ or asymptomatic $(\mathrm{n}=71)$, and underwent conventional coronary angiography (CAG). For CTCA we administered an IV bolus of $100 \mathrm{ml}$ of iodinated contrast material. CT-CA was compared to CAG using a threshold for significant stenosis of $\geq 50 \%$. The prevalence of disease dem- onstrated at CAG was $15 \%(1.4 \%$ in asymptomatic). The population at $\mathrm{CAG}$ showed no or non-significant disease in $85 \%(238 / 279)$, single vessel disease in $9 \%(25 / 279)$, and multi-vessel disease in $6 \%(16 / 279)$. Sensitivity, specificity, and positive and negative predictive values of CT-CA vs. CAG on the patient level were $100 \%, 95 \%, 76 \%$, and $100 \%$ in the overall population and $100 \%$, $100 \%, 100 \%$, and $100 \%$ in asymptomatic patients, respectively. CT-CA proves high diagnostic performance in patients with or without symptoms and with zero CACS. The prevalence of significant disease detected by CT-CA was not negligible in asymptomatic patients. The role of CT-CA in asymptomatic patients remains uncertain.

Keywords Multidetector computed tomography · Conventional coronary angiography $\cdot$ Coronary artery disease $\cdot$ Calcium score . Asymptomatic

\section{Introduction}

Computed tomography coronary angiography (CT-CA) is a robust imaging modality that allows non-invasive detection of significant coronary stenoses [1-5]. Several studies have demonstrated a good diagnostic performance of CT-CA using different generations of CT equipment [1-5]. Sixtyfour-slice CT is the current standard for CT-CA, and with such machines, CT-CA can explore the entire coronary tree
$[4,5]$. CT-CA has a high negative predictive value, and it is reliable for significant coronary artery disease (CAD) exclusion.

The Coronary Calcium Score (CACS) has been developed and widely used for years as a tool for cardiovascular risk stratification, and its incremental value over conventional risk factors has been demonstrated [6-8]. Studies and guidelines claim that with zero CACS, the likelihood of obstructive CAD and/or cardiovascular coronary events is 
very low [6-9]. Recent reports have challenged this finding reporting a non-negligible prevalence of obstructive $\mathrm{CAD}$ in patients with zero CACS $[10,11]$.

The aim of this study was to assess the diagnostic accuracy of CT-CA in patients with zero CACS vs. conventional coronary angiography $(\mathrm{CAG}$, assumed to be the gold standard) in a population of patients with and without symptoms.

\section{Materials and methods}

Subjects

During a period of 12 months we enrolled 279 consecutive patients with typical/atypical chest pain (symptomatic= 208 ) and without symptoms (asymptomatic $=71$; enrolled based on risk profile and/or stress test abnormalities) with zero CACS (96 men and 183 women; mean age \pm SD: $48 \pm$ 12 years, range: $21-77$ years) who underwent CT-CA followed by CAG. Patients in sinus heart rhythm capable of holding their breath for $12 \mathrm{~s}$ and without contraindications to the administration of iodinated contrast agents were included. Exclusion criteria were previous bypass graft surgery or percutaneous coronary intervention with stent implantation. The study was approved by the local medical ethics committee, and all patients gave written informed consent (Table 1).

\section{Patient preparation before CT}

Patients with heart rates above $65 \mathrm{bpm}$ received a single intravenous dose of $5 \mathrm{mg}$ atenolol $10 \mathrm{~min}$ before CT-CA under ECG and blood pressure control. Immediately before CT-CA we administered sub-lingual isosorbide dinitrates $(0.3 \mathrm{mg})$ to all patients.

\section{CT-CA protocol}

All patients underwent CT coronary angiography using 64slice CT (Sensation 64 Cardiac $^{\circledR}$, Siemens, Forchheim, Germany). For the unenhanced CT, a standard protocol described elsewhere was applied $[1-5,12]$. The assessment of CACS was performed with a dedicated software (CaScore ${ }^{\circledR}$, Siemens, Germany), and an Agatston Calcium Score of zero was used to enroll patients [13].

A bolus of $100 \mathrm{ml}$ contrast material (iomeprol, Iomeron ${ }^{\circledR} 400$, Bracco, Milan, Italy) was injected into the antecubital vein (flow rate of $5 \mathrm{ml} / \mathrm{s}$ ) and the CT-CA triggered with a bolus tracking technique with a region of

Table 1 Demographics

\begin{tabular}{llll}
\hline & Total & Symptomatic & Asymtomatic \\
\hline Population & & & \\
Number of patients & 279 & 208 & 71 \\
Age (mean \pm SD; range) & $48 \pm 12(21-77)$ & $48 \pm 11(22-77)$ & $47 \pm 13(21-68)$ \\
Gender (M/F) & $96 / 183$ & $57 / 151$ & $39 / 32^{*}$ \\
Symptoms & & & $0(0)^{*}$ \\
Typical angina (\%) & $99(36)$ & $99(48)$ & $0(0)^{*}$ \\
Atypical chest pain (\%) & $87(31)$ & $87(42)$ & $0(0)^{*}$ \\
Unstable angina (\%) & $7(3)$ & $7(3)$ & $0(0)^{*}$ \\
AMI (\%) & $15(5)$ & $15(7)$ & $71(100)^{*}$ \\
Asymptomatic (\%) & $71(25)$ & $0(0)$ & $31(44)$ \\
Cardiovascular risk factors & & & $18(25)$ \\
Hypertension (\%) & $101(36)$ & $70(34)$ & $4(6)$ \\
Dyslipidaemia (\%) & $101(36)$ & $83(40)$ & $19(27)$ \\
Diabetes (\%) & $18(7)$ & $14(7)$ & $20(28)$ \\
Smoking (\%) & $71(25)$ & $52(25)$ & $27 \pm 4(19-35)$ \\
Family history (\%) & $82(29)$ & $62(30)$ & $1.4 \pm 0.8$ \\
BMI (kg/m $;$ mean \pm SD; range) & $28 \pm 5(19-42)$ & $29 \pm 5(20-42)$ & $1.7 \pm 1.1$ \\
RF/pt (mean \pm SD) & $1.7 \pm 1.0$ & & \\
\hline
\end{tabular}

$* \mathrm{p}<0.05$

Abbreviations: $\mathrm{SD}=$ Standard deviation; $\mathrm{M} / \mathrm{F}=$ male/female; $\mathrm{BMI}=$ body mass index; $\mathrm{RF} / \mathrm{pt}=$ risk factors per patient; $\mathrm{AMI}=$ acute myocardial infarction 
interest placed on the ascending aorta (threshold +100 $\mathrm{HU})$.

Radiation exposure associated to this CT-CA protocol, calculated using dedicated software (WinDose ${ }^{\mathbb{R}}$, Institute of Medical Physics, Erlangen, Germany), was 15-21 (female-male) $\mathrm{mSv}$.

\section{CT image reconstruction}

A retrospective ECG-gated technique was used for the reconstruction of images. Data acquired during a single heartbeat were used. Datasets were reconstructed during the mid-to-end diastolic phase, with reconstruction windows set at -300 to $-450 \mathrm{~ms}$ before the next Rwave or $60 \%$ to $70 \%$ of the R-R interval. In case of insufficient image quality, additional reconstructions during the end-systolic phase $(25 \%$ to $35 \%$ of the R-R interval) were performed. The reconstructed slice thickness was $0.75 \mathrm{~mm}$ with an increment of $0.4 \mathrm{~mm}$. All the CT datasets were filtered with a medium-soft convolution kernel.

The CT images were scored by a consensus of two experienced readers (one with 9 years of experience and one with 4 years of experience), blinded of the results of conventional coronary angiography.

Maximum intensity projections were used in order to provide an anatomical overview of the coronary arteries and to identify side branches, and multi-planar reformatted images were used to classify lesions as significant or nonsignificant.

\section{Quantitative coronary angiography (QCA)}

All coronary segments visualized at catheterization were included for comparison with CT. The segments were classified according to a modified 17-segment American Heart Association (AHA) model [14]. A single observer blinded to the $\mathrm{CT}$ results quantitatively evaluated the coronary lumen of all coronary segments using dedicated software $\left(\mathrm{CAAS}^{\circledR}\right.$, Pie Medical, Maastricht, The Netherlands). Stenoses were classified as significant in case of a mean lumen diameter reduction of $\geq 50 \%$ in two orthogonal projections.

\section{Statistics}

Continuous variables are expressed as mean values $( \pm \mathrm{SD})$. Differences between groups were compared using the Student's $t$, chi-square, and Mann-Whitney tests, as appropriate. With quantitative coronary angiography (QCA) as the standard of reference, to determine the diagnostic accuracy of CT-CA coronary angiography for the detection of significant lesions in coronary arteries, we calculated diagnostic accuracy as sensitivity, specificity, positive and negative predictive value, positive (Sensitivity/[1-Specificity]) and negative ([1-Sensitivity]/Specificity]) likelihood ratios with the corresponding 95\% central confidence intervals (calculated with binomial expansion).

We performed ROC analysis and calculated the area under the curve (AUC). Diagnostic accuracy was calculated on a per-segment and per-patient (presence or absence of any lesions in each individual patient) basis. Graphs of conditional probabilities were plotted.

All statistics were performed in the entire population and in the sub-groups with and without symptoms (symptomatic and asymptomatic, respectively). All statistics were performed using SPSS 12.0 software.

\section{Results}

Prevalence of disease

Overall, 82 significant lesions were detected by CAG, resulting in a prevalence of 0.38 lesions per patient (Table 2). CAG revealed no significant stenoses in 238 $(85 \%)$ patients, and at least one significant stenosis in 41 $(15 \%)$ patients.

\section{Total population}

Intra-venous atenolol was administered in $85.3 \%$ (203/238) of the patients. As a result, the average heart rate during the scan was $59.4 \pm 6.7 \mathrm{bpm}$. Average acquisition time to obtain the CT-CA data was $10 \pm 2 \mathrm{~s}$ (range, 9-13 s). No significant adverse reactions to contrast material were recorded (Table 3, Graphs 1 and 2).

A total of 4,097 coronary segments were available. Eighty-two significant stenoses were present, and 78 were correctly identified by CT-CA. Four significant stenoses were missed by CT-CA mainly due to misjudgment in more distal branches (two diagonal branches, one marginal branch and one posterior descending artery) with smaller diameters. Twenty-three segments were judged false positive on CT-CA as compared to CAG. The main reason for overestimation was residual motion artifacts (18/23).

Sensitivity, specificity, and positive and negative predictive values on the segment level were $95 \%, 99 \%$, $77 \%$, and $100 \%$ in the overall population.

On a per-patient level, all 41 patients with at least one significant stenosis were correctly identified by CT-CA. Inter-observer agreement was high (weighted kappa= 0.84). The area under the curve calculated in the ROC analysis was 0.973 .

Sensitivity, specificity, and positive and negative predictive values on the patient level were $100 \%, 95 \%$, $76 \%$, and $100 \%$ in the overall population. 
Table 2 Distribution of coronary artery disease as detected by conventional coronary angiography

\begin{tabular}{|c|c|c|c|}
\hline Parameters & Total & Symptomatic & Asymtomatic \\
\hline Number of patients & 279 & 208 & 71 \\
\hline No disease/non-significant disease $(\%)$ & $238(85.3)$ & $168(80.8)$ & $70(98.6)$ \\
\hline Single-vessel disease (\%) & $25(8.9)$ & $24(11.5)$ & $1(1.4)$ \\
\hline Dual-vessel diasease $(\%)$ & $8(2.9)$ & $8(3.9)$ & $0(0)$ \\
\hline Triple-vessel disease (\%) & $8(2.9)$ & $8(3.9)$ & $0(0)$ \\
\hline Multi-vessel diasease $(\%)$ & $16(5.8)$ & $16(7.7)$ & $0(0)$ \\
\hline Total disease $(\%)$ & $41(14.7)$ & $40(19.2)$ & $1(1.4)^{*}$ \\
\hline
\end{tabular}

$* \mathrm{p}<0.05$

Symptomatic vs. asymptomatic

The prevalence of disease in symptomatic and asymptomatic patients was $19.2 \%$ and $1.4 \%$, respectively (Table 3 , Graphs 1 and 2).

Sensitivity, specificity, and positive and negative predictive values on a per-patient level were $100 \%$ (91-100), 92\% (87-95), 76\% (61-86), and 100\% (97$100)$ in symptomatic patients and $100 \%(2-100), 100 \%$ $(94-100), \quad 100 \% \quad(2-100)$, and $100 \%(94-100)$ in asymptomatic patients, respectively $(\mathrm{p}<0.05)$. In both groups, all patients with at least one significant stenosis were correctly identified. Inter-observer agreement was high in symptomatic and asymptomatic patients (weighted kappa $=0.82$ and 1.00 , respectively). The area under the curve calculated in the ROC analysis was 0.96 and 1.00 for symptomatic and asymptomatic, respectively.

\section{Discussion}

The results of our study show an optimal diagnostic performance of CT-CA in patients with zero CACS. All patients with at least one significant coronary artery stenosis were correctly identified by CT-CA. The diagnostic performance of CT-CA was higher $(100 \%$ sensitivity and $100 \%$ specificity) in asymptomatic patients even though the prevalence of disease in the population was very low $(1.4 \% ; 1 / 71)$. In this population, the negative

Table 3 Diagnostic accuracy of CT-CA vs. CAG

\begin{tabular}{|c|c|c|c|c|c|c|c|c|c|c|c|c|c|}
\hline & & No. & $\mathrm{TP}$ & $\mathrm{TN}$ & FP & $\mathrm{FN}$ & Sens. & Spec. & PPV & NPV & Prev. & $\mathrm{LR}+$ & LR- \\
\hline \multicolumn{14}{|l|}{ Total } \\
\hline \multirow[t]{2}{*}{ CT-CA } & Per-segment & 4097 & 78 & 3992 & 23 & 4 & $\begin{array}{l}95.12 \\
(87-98)\end{array}$ & $\begin{array}{l}99.42 \\
(99-99)\end{array}$ & $\begin{array}{l}77.2 \\
(67-84)\end{array}$ & $\begin{array}{l}99.9 \\
(99-99)\end{array}$ & 2 & $\begin{array}{l}166 \\
(110-250)\end{array}$ & $\begin{array}{l}0.049 \\
(0.018-0.12)\end{array}$ \\
\hline & Per-patient & 279 & 41 & 225 & 13 & 0 & $\begin{array}{l}100 \\
(91-100)\end{array}$ & $\begin{array}{l}94.53 \\
(90-97)\end{array}$ & $\begin{array}{l}75.9 \\
(62-86)\end{array}$ & $\begin{array}{l}100 \\
(98-100)\end{array}$ & 14.69 & $\begin{array}{l}18.3 \\
(10.8-31.1)\end{array}$ & $0(0-\mathrm{NA})$ \\
\hline \multicolumn{14}{|c|}{ Symptomatic } \\
\hline \multirow[t]{2}{*}{ CT-CA } & Per-segment & 3090 & 77 & 2986 & 23 & 4 & $\begin{array}{l}95.1 \\
(87-98)\end{array}$ & $\begin{array}{l}99.2 \\
(98-99)\end{array}$ & $\begin{array}{l}77 \\
(67-84)\end{array}$ & $\begin{array}{l}99.9 \\
(99-99)\end{array}$ & 2.6 & $\begin{array}{l}124 \\
(82.5-187.4)\end{array}$ & $\begin{array}{l}0.049 \\
(0.019-0.12)\end{array}$ \\
\hline & Per-patient & 208 & 40 & 155 & 13 & 0 & $\begin{array}{l}100 \\
(91-100)\end{array}$ & $\begin{array}{l}92.3 \\
(87-95)\end{array}$ & $\begin{array}{l}75.5 \\
(61-86)\end{array}$ & $\begin{array}{l}100 \\
(97-100)\end{array}$ & 19.23 & $\begin{array}{l}12.92 \\
(7.7-21.8)\end{array}$ & 0 (0-NA) \\
\hline \multicolumn{14}{|c|}{ Asymptomatic } \\
\hline \multirow[t]{2}{*}{ CT-CA } & Per-segment & 1007 & 1 & 1006 & 0 & 0 & $\begin{array}{l}100 \\
(2-100)\end{array}$ & $\begin{array}{l}1000 \\
(99-100)\end{array}$ & $\begin{array}{l}100 \\
(2-100)\end{array}$ & $\begin{array}{l}100 \\
(99-100)\end{array}$ & 0.09 & NA & 0 (0-NA) \\
\hline & Per-patient & 71 & 1 & 70 & 0 & 0 & $\begin{array}{l}100 \\
(2-100)\end{array}$ & $\begin{array}{l}100 \\
(94-100)\end{array}$ & $\begin{array}{l}100 \\
(2-100)\end{array}$ & $\begin{array}{l}100 \\
(94-100)\end{array}$ & 1.4 & NA & $0(0-\mathrm{NA})$ \\
\hline
\end{tabular}

The values of diagnostic accuracy are shown for the entire population and for the subgroups of patients with and without symptoms. The criteron used for comparison with CT-CA is lumen reduction $\geq 50 \%$ on CAG. Diagnostic accuracy is expressed in percentage with $95 \%$ confidence intervals calculated with binomial expansion. Prevalence of disease is expressed in percentage. Positive and negative likelihood ratios are shown with $95 \%$ confidence intervals calculated with binomial expansion.

Abbreviations: $\mathrm{TP}=$ true positive; $\mathrm{TN}=$ true negative; $\mathrm{FP}=$ false positive; $\mathrm{FN}=$ false negative; $\mathrm{PPV}=$ positive predictive value; $\mathrm{NPV}=$ negative predictive value; Prev. $=$ prevalence of disease; $\mathrm{LR}+=$ positive likelihood ratio; LR- = negative likelihood ratio; $\mathrm{CT}-\mathrm{CA}=\mathrm{CT}$ coronary angiography; $\mathrm{NA}=$ not assessable 
Diagnostic Accuracy - per patient

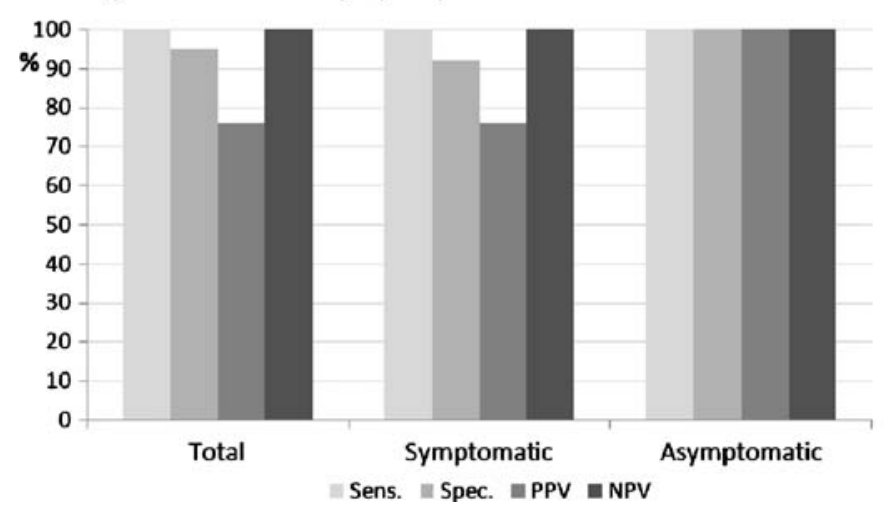

Graph 1 Bar graph of diagnostic accuracy. The bar graph shows diagnostic accuracy for CT-CA as compared to CAG in the total population, in symptomatic and asymptomatic patients, on a perpatient (left panel) and per-segment (right panel) level. CT-CA shows better diagnostic performance in asymptomatic patients both

predictive value of CACS alone in this group would have been $98.6 \%$. However, the patient with obstructive CAD would not have been detected (sensitivity $0 \%$ ).

In this regard, we have to account for the impact of prevalence of disease and conditional probabilities. The population presented is unique, since it has a very low pretest likelihood and prevalence of disease. The good capability of CT-CA to identify correctly the very few patients requiring $\mathrm{CAG}$ within this population is counterbalanced by a false-positive rate of $6.3 \%(13 / 208)$ in symptomatic patients and $0 \%(0 / 71)$ in asymptomatic patients. The rate of unnecessary CAG would have been $4.7 \%(13 / 279)$ in the total population.

Kelly et al. reported a population of 325 patients (high risk for CAD or atypical symptoms or abnormal stress test results) with zero CACS undergoing CT-CA and CAG [10]. The authors found 167 patients with non-calcified plaques and $18(5.5 \%)$ patients with obstructive CAD [10]. They concluded that an atherosclerotic burden and
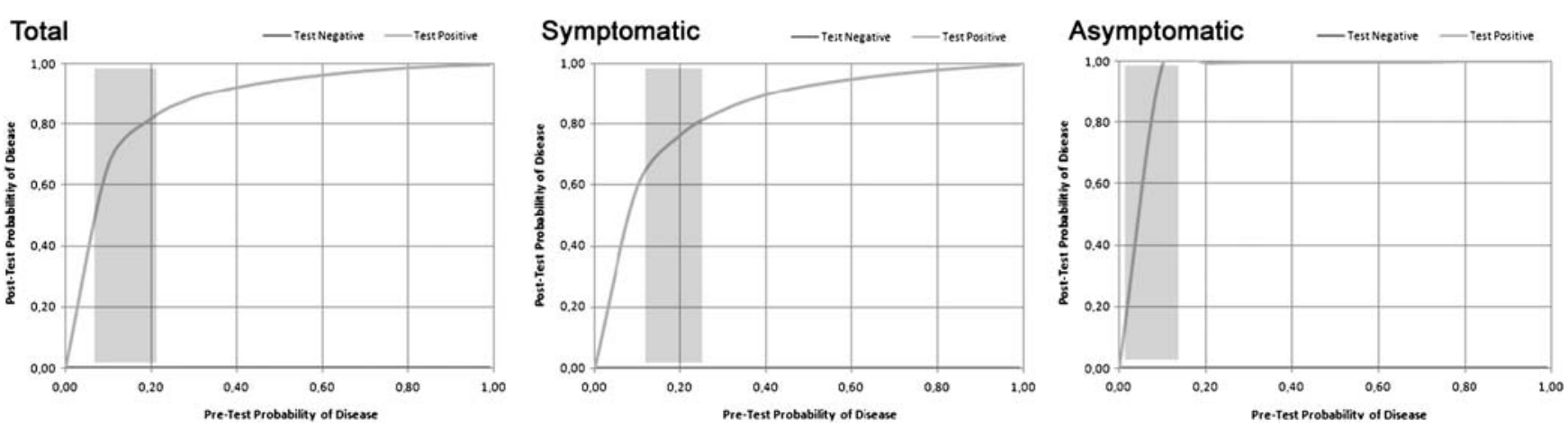

Graph 2 Graphs of conditional probabilities. The graph shows the conditional probabilities for CT-CA as the relationship between pretest and post-test probability of disease. The grey bars highlight the points corresponding to the prevalence of disease for the individual populations and sub-groups. The post-test negative probability (test

negative - dark gray line) of CT-CA is always very high. The posttest positive probability (test positive- bright gray line) is high for the total population and for symptomatic patients, while for asymptomatic patients it is very high. Abbreviations: $\mathrm{CT}-\mathrm{CA}=\mathrm{CT}$ coronary angiography 
had non-calcified plaques, with $1.8 \%(15 / 825)$ having significant or severe obstructive CAD. They concluded that the presence of occult CAD is not negligible in the asymptomatic population.

From our study and from the ancillary literature described, we can extrapolate that there is a not negligible obstructive burden of disease in populations with zero CACS and that this prevalence is very much affected by clinical presentation (symptomatic vs. asymptomatic).

As demonstrated by Mowatt et al. in a cost-effectiveness analysis, the best strategies for detection of obstructive $\mathrm{CAD}$ in populations with prevalence of disease around $10 \%$ are those with CT-CA in the first or second place (with stress ECG in the first place) within the diagnostic algorithm [16]. In this analysis the authors included stress ECG, CT-CA, CAG, and stress SPECT [16]. The costeffectiveness analysis does not take into account the impact of radiation dose. However, even stress SPECT and CAG carry a significant burden of radiation.

The main issue concerning the use of CT-CA in patients with zero CACS is related to radiation dose. Current standards in CT-CA provide doses that can range from $8 \mathrm{mSv}$ to $18 \mathrm{mSv}$ (mean=12 $\mathrm{mSv}$ ) as reported by Hausleiter et al. in a very large worldwide survey [17]. With this burden of radiation dose, the implementation of CT-CA in patients with zero CACS is very questionable.

Recently, newer CT hardware and software solutions have been introduced, allowing to scan the coronary arteries with much lower radiation exposure. In particular, the implementation of prospective ECG triggering for CT-
CA allows completing the investigation with $1-5 \mathrm{mSv}$, depending on which technology is applied [18-20]. These improvements will probably lead to reconsideration of the indications of CT-CA.

\section{Limitations}

Firstly, the population is relatively small, especially for the sub-analysis of asymptomatic patients $(\mathrm{n}=71)$, and this results in a low absolute number of patients with obstructive CAD. However, all patients underwent CAG, and validation was granted; the relative prevalence is in line with other larger studies $(1.4 \%$ in our study and $1.8 \%$ in the study by Choi et al.) [15].

Secondly, the radiation dose in asymptomatic patients remains a matter of concern, as discussed above.

\section{Conclusions}

CT-CA proves optimal diagnostic performance in symptomatic and asymptomatic patients with zero CACS. CACS remains a safer and reliable tool for the exclusion of disease in asymptomatic patients. Concerns are related to the risk of radiation dose vs. the benefits of a correct diagnosis. Newer hardware and software improvements bringing a consistent reduction in radiation dose will make the option of CT-CA more feasible in this population of patients.

\section{References}

1. Nieman K, Oudkerk M, Rensig BJ et al (2001) Coronary angiography with multislice computed tomography. Lancet 357:599-603

2. Mollet NR, Cademartiri F, Krestin GP et al (2005) Improved diagnostic accuracy with 16-row multi-slice computed tomography coronary angiography. $\mathrm{J}$ Am Coll Cardiol 45:128-132

3. Mollet NR, Cademartiri F, Nieman K et al (2004) Multislice spiral ct coronary angiography in patients with stable angina pectoris. J Am Coll Cardiol 43:2265-2270

4. Mollet NR, Cademartiri F, van Mieghem CA et al (2005) High-resolution spiral computed tomography coronary angiography in patients referred for diagnostic conventional coronary angiography. Circulation 112:2318-2323
5. Cademartiri F, Malagutti P, Belgrano M et al (2005) Non-invasive coronary angiography with 64-slice computed tomography. Minerva Cardioangiol 53:465-472

6. Shaw LJ, Raggi P, Schisterman E, Berman DS, Callister TQ (2003) Prognostic value of cardiac risk factors and coronary artery calcium screening for all-cause mortality. Radiology 228:826-833

7. Greenland P, LaBree L, Azen SP, Doherty TM, Detrano RC (2004) Coronary artery calcium score combined with Framingham score for risk prediction in asymptomatic individuals. Jama 291:210-215

8. Greenland P, Smith SC Jr, Grundy SM (2001) Improving coronary heart disease risk assessment in asymptomatic people: role of traditional risk factors and noninvasive cardiovascular tests. Circulation 104:1863-1867
9. Oudkerk M, Stillman AE, Halliburton SS et al (2008) Coronary artery calcium screening: current status and recommendations from the European Society of Cardiac Radiology and North American Society for Cardiovascular Imaging. Eur Radiol 18:2785-2807

10. Kelly JL, Thickman D, Abramson SD et al (2008) Coronary CT angiography findings in patients without coronary calcification. AJR Am J Roentgenol 191:50-55

11. Akram K, Voros S (2008) Influence of symptoms and age on the predictive value of coronary artery calcium scanning. J Am Coll Cardiol 52:2214 author reply 2214

12. Cademartiri F, Runza G, La Grutta L et al (2005) Non-invasive evaluation of coronary calcium. Radiol Med (Torino) 110:506-522 
13. Agatston AS, Janowitz WR, Hildner FJ, Zusmer NR, Viamonte M Jr, Detrano R (1990) Quantification of coronary artery calcium using ultrafast computed tomography. J Am Coll Cardiol 15:827-832

14. Austen WG, Edwards JE, Frye RL et al (1975) A reporting system on patients evaluated for coronary artery disease. Report of the Ad Hoc Committee for Grading of Coronary Artery Disease, Council on Cardiovascular Surgery, American Heart Association. Circulation 51:5-40
15. Choi EK, Choi SI, Rivera JJ et al (2008) Coronary computed tomography angiography as a screening tool for the detection of occult coronary artery disease in asymptomatic individuals. J Am Coll Cardiol 52:357-365

16. Mowatt G, Cummins E, Waugh $\mathrm{N}$ et al (2008) Systematic review of the clinical effectiveness and cost-effectiveness of 64-slice or higher computed tomography angiography as an alternative to invasive coronary angiography in the investigation of coronary artery disease. Health Technol Assess 12:iii-iv ix-143

17. Hausleiter J, Meyer T, Hermann F et al (2009) Estimated radiation dose associated with cardiac CT angiography. Jama 301:500-507
18. Hirai N, Horiguchi J, Fujioka C et al (2008) Prospective versus retrospective ECG-gated 64-detector coronary CT angiography: assessment of image quality, stenosis, and radiation dose. Radiology 248:424-430

19. Stolzmann P, Leschka S, Scheffel H et al (2008) Dual-source CT in step-andshoot mode: noninvasive coronary angiography with low radiation dose. Radiology 249:71-80

20. Scheffel H, Alkadhi H, Leschka S et al (2008) Low-dose CT coronary angiography in the step-and-shoot mode: diagnostic performance. Heart 94:1132-1137 